\title{
A Lower Bound for the Norm of the Theta Operator*
}

\author{
By L. Alayne Parson
}

\begin{abstract}
The Poincare theta operator maps the space of holomorphic functions with period one onto the space of cusp forms for a finitely generated Fuchsian group. It is easy to show that the norm of the operator does not exceed one. In the case of the classical modular group and weight six, it is now shown that the norm is bounded below by .927 .
\end{abstract}

1. Definition of the Theta Operator. Let $\Gamma$ be a finitely generated Fuchsian group acting on the upper half plane $\mathcal{H}$ and containing the translation $S z=z+1$. Let $A_{q}(\Gamma)$ be the space of cusp forms of weight $q . A_{q}(\Gamma)$ is then a Banach space with norm

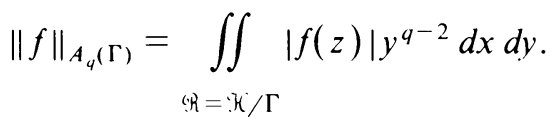

When $\Gamma=\Gamma_{\infty}=\langle S\rangle$, denote $A_{q}\left(\Gamma_{\infty}\right)$ by $A_{q}$. The Poincare $\theta$-operator, $\theta_{q}$, which is defined by

$$
\theta_{q}(f)=\sum_{A \in \Gamma / \Gamma_{\infty}} f(A z) A^{\prime q}(z)
$$

is a surjective continuous linear operator from $A_{q}$ to $A_{q}(\Gamma)$. That $\left\|\theta_{q}\right\| \leqslant 1$ is trivial. (See, for instance, Kra [1, Chapter 3].) Although many people suspect that $\left\|\theta_{q}\right\|<1$ for various choices of $q$ and $\Gamma$, this is not known, one way or another, in even a single case. Also, the standard lower bound on the norm is $\left\|\theta_{q}\right\| \geqslant(q-1) /(2 q-1)$ (see Kra [1, p. 91]).

2. The Modular Group and Weight Six. The purpose of this note is to improve the lower bound on the theta operator norm for the modular group $\Gamma(1)$ and weight $q=6$. In this case it is shown that $\left\|\theta_{6}\right\| \geqslant .927$, which is a marked improvement on the standard lower bound of 5/11. $A_{6}(\Gamma(1))$ is the one-dimensional space of modular cusp forms which is spanned by the famous discriminant function

$$
\Delta(z)=e^{2 \pi i z} \prod_{n=1}^{\infty}\left(1-e^{2 \pi i n z}\right)^{24} .
$$

The Fourier expansion at $\infty$ is $\Delta(z)=\sum_{n=1}^{\infty} \tau(n) e^{2 \pi i n z}$, where $\tau(n)$ is the celebrated Ramanujan tau function.

To improve the lower bound on $\left\|\theta_{6}\right\|$ it is necessary to evaluate $\left\|\theta_{6}(f)\right\|_{A_{6}(\Gamma(1))} /\|f\|_{A_{6}}$ for various choices of $f$ for which both $\left\|\theta_{6}(f)\right\|_{A_{6}(\Gamma(1))}$ and $\|f\|_{A_{6}}$ are easy to evaluate accurately. Specifically, use is made of the following result.

Received July 14, 1982.

1980 Mathematics Subject Classification. Primary 10D12, 30F35.

*Research partially supported by NSF grant MCS-8202025. 
THEOREM. Set $p_{k}(z)=\sum_{n=0}^{k} a_{n} e^{2 \pi i n z}$ and

$$
f_{k}(z)=e^{2 \pi i z} p_{k}^{2}(z)=\sum_{n=1}^{2 k+1} b_{n} e^{2 \pi i n z} .
$$

Then

$$
\left\|f_{k}\right\|_{A_{h}}=\frac{4 !}{(2 \pi)^{5}} \sum_{n=0}^{k} \frac{\left|a_{n}\right|^{2}}{(1+2 n)^{5}}
$$

and

$$
\left\|\theta_{6}\left(f_{k}\right)\right\|_{A_{6}(\Gamma(1))}=\frac{10 !\|\Delta\|_{A_{6}(\Gamma(1))}}{(4 \pi)^{11}(\Delta, \Delta)}\left|\sum_{n=1}^{2 k+1} \frac{b_{n} \tau(n)}{n^{11}}\right|,
$$

where $(\Delta, \Delta)=\iint_{\mathcal{K} / \Gamma(1)}|\Delta(z)|^{2} y^{10} d x d y$ is the Petersson scalar product of $\Delta(z)$ with itself.

Proof. Since

$$
\left\|f_{k}\right\|_{A_{6}}=\int_{0}^{x} \int_{0}^{1}\left|f_{k}(z)\right| y^{4} d x d y=\int_{0}^{x} \int_{0}^{1} e^{2 \pi y}\left|p_{k}(z)\right|^{2} y^{4} d x d y
$$

an application of Parseval's formula gives that

$$
\left\|f_{k}\right\|_{A_{6}}=\int_{0}^{\infty} e^{2 \pi y^{\prime}} y^{4} \sum_{n=0}^{h}\left|a_{n}\right|^{2} e^{4 \pi n y} d y=\frac{4 !}{(2 \pi)^{5}} \sum_{n=0}^{h} \frac{\left|a_{n}\right|^{2}}{(2 n+1)^{5}} .
$$

Next note that $\theta_{6}\left(f_{k}\right)=\sum_{n}^{2 k+1}{ }_{1} b_{n} \theta_{6}\left(e^{2 \pi i n z}\right)$. However, since $A_{6}(\Gamma(1))$ has dimension one, $\theta_{6}\left(e^{2 \pi i n z}\right)=c_{n} \Delta$, where $c_{n}$ can be determined easily using properties of the scalar product. $\theta_{6}\left(e^{2 \pi i n z}\right)$ is generally denoted by $G_{n}$, the $n$th Poincare series. Then $\left(G_{n}, \Delta\right)=\left(c_{n} \Delta, \Delta\right)$ and $c_{n}=\left(G_{n}, \Delta\right) /(\Delta, \Delta)$. However, by the scalar product formula (Rankin [5, p. 149]),

$$
\left(G_{n}, \Delta\right)=\frac{10 ! \tau(n)}{(4 \pi n)^{11}}
$$

As a result

$$
\left\|\theta_{6}\left(f_{k}\right)\right\|_{A_{h}(\Gamma(1))}=\frac{10 !\|\Delta\|_{A_{6}(\Gamma(1))}}{(4 \pi)^{11}(\Delta, \Delta)}\left|\sum_{n=1}^{2 h+1} \frac{b_{n} \tau(n)}{n^{11}}\right| .
$$

In order to use the above results it is necessary to know the values of $(\Delta, \Delta)$ and $\|\Delta\|_{A_{6}(\Gamma(1))}$. Lehmer [2] has shown that

$$
(\Delta, \Delta)=10^{-6} \times 1.03529048 .
$$

$\|\Delta\|_{A_{6}(\Gamma(1))}$ was evaluated at Ohio State on the Amdahl 470 using a nested Simpson's rule in double-precision Fortran giving

$$
\|\Delta\|_{A_{6}(\Gamma(1))}=.00070225689 .
$$

3. The Lower Bound. Now consider polynomials $p_{k}(z)$ with $a_{0} \neq 0$. For $k=1$ to 30 , the approximate maximum of $\left\|\theta_{6}\left(f_{k}\right)\right\|_{A_{6}(\Gamma(1))} /\left\|f_{k}\right\|_{A_{6}}$ was calculated, where the coefficients $a_{1}, \ldots, a_{k}$ of $p_{k}(z)$ were taken to be real. The results are listed in Table I and show that $\left\|\theta_{6}\right\| \geqslant .927$. All calculations were carried out in basic on Apple $\mathrm{II}^{+}$. 
The approximate maximum was found using a Hooke-Jeeves pattern search method as implemented by Nash in [3] with tolerance .001 . These calculations were verified for $k=5,10,15,20$ using the Nelder-Meade simplex method as coded by O'Neill in [4] and were found to be in excellent agreement.

TABLE I

\begin{tabular}{|c|c|}
\hline$k$ & approximate maximum of $\left\|\theta_{6}\left(f_{k}\right)\right\|_{A_{6}(\Gamma(1))} /\left\|f_{k}\right\|_{A_{6}}$ \\
\hline 1 & .852608215 \\
\hline 2 & .881141356 \\
\hline 3 & .893379912 \\
\hline 4 & .893629489 \\
\hline 5 & .903739297 \\
\hline 6 & .909550921 \\
\hline 7 & .910936172 \\
\hline 8 & .913052751 \\
\hline 9 & .913054225 \\
\hline 10 & .913458802 \\
\hline 11 & .916965021 \\
\hline 12 & .916981747 \\
\hline 13 & .919648617 \\
\hline 14 & .920731796 \\
\hline 15 & .921503011 \\
\hline 16 & .922274684 \\
\hline 17 & .922296736 \\
\hline 18 & .922403535 \\
\hline 19 & .922519335 \\
\hline 20 & .924591551 \\
\hline 21 & .926187880 \\
\hline 22 & .926225627 \\
\hline 23 & .926568015 \\
\hline 24 & .926636356 \\
\hline 25 & .926636555 \\
\hline 26 & .926643190 \\
\hline 27 & .926922205 \\
\hline 28 & .927056661 \\
\hline 29 & .927062756 \\
\hline 30 & .927068371 \\
\hline
\end{tabular}

Department of Mathematics

The Ohio State University

Columbus, Ohio 43210

1. I. KRA, A utomorphic Forms and Kleinian Groups, Benjamin, Reading, Mass., 1972.

2. D. H. Lehmer, “Ramanujan's function $\tau(n)$," Duke Math J., v. 10, 1943, pp. 483-492.

3. J. C. NASH, “Function minimizations,” Interface Age, v. 7, 1982, pp. 34-42.

4. R. O’NeILl, "Function minimization using a simplex procedure," Applied Statistics, v. 20, 1971, pp. $338-345$.

5. R. Rankin, Modular Forms and Functions, Cambridge Univ. Press, Cambridge, 1977. 